\title{
État et perspectives de lutte contre Sitotroga cerealella (Olivier, 1789), déprédateur des céréales au Bénin : synthèse bibliographique.
}

\section{REVIEW PAPER}

\author{
ADJALIAN Elvis ${ }^{1,2}$, NOUDOGBESSI Jean-Pierre ${ }^{1}$, KOSSOU Dansou², SOHOUNHLOUE Dominique ${ }^{*}$ \\ ${ }^{1}$ Laboratoire d'Étude et de Recherche en Chimie Appliquée (LERCA) École Polytechnique d'Abomey- \\ Calavi/Université d'Abomey-Calavi 01 BP 2009 Cotonou, R. Bénin. \\ 2Laboratoire de Production Végétale ; Faculté des Sciences Agronomiques ; Université d'Abomey-Calavi ;01 B.P 526 \\ Cotonou, Bénin ; 06 BP 258 Akpakpa, Cotonou, R. Bénin \\ * Auteur pour correspondance : ksohoun@bj.refer.org, csohoun@gmail.com
}

Original submitted in on $30^{\text {th }}$ April 2014. Published online at www.m.elewa.org on $31^{\text {st }}$ July 2014. http://dx.doi.org/10.4314/jab.v79i1.16

\begin{abstract}
RESUME
Objectif: Le présent article de revue fait état des études relatives aux différentes méthodes de lutte contre Sitotroga cerealella (alucite des céréales) et met l'accent sur l'utilisation des substances naturelles en perspective de lutte contre ce déprédateur. $S$. cerealella est l'un des ravageurs les plus redoutables dans les systèmes de conservation traditionnels en Afrique et particulièrement au Bénin.

Méthodologie et Résultats: Dans la recherche des informations sur les luttes contre l'alucite des céréales, plusieurs travaux scientifiques publiés ont été consultés et leur synthèse a été faite. L'examen des résultats de recherche sur le contrôle de ce déprédateur de plusieurs céréales a révélé l'utilisation fréquente de produits chimiques de synthèse comme la deltaméthrine, le malathion et la phosphine seuls ou combinés en fumigation surtout. Plusieurs chercheurs se sont intéressés, dans le cadre des luttes physiques, aux irradiations ionisantes provenant de source de rayons gamma du cobalt 60 et des variétés de céréales résistantes dans le cas du riz, du blé ou de maïs. Aussi, des parasites, pathogènes ou prédateurs (Trichogramma spp, Blattisocius tarsalis, Cotesia ruficrus, Pteromalus cerealella et Bracon hebetor) ont-ils été expérimentés comme insecticide biologique sur différents stades de développement de $S$. cerealella. Les traitements à base de poudres ou d'extraits de plantes à potentialités insecticides ou insectifuges tels que ceux de Cymbopogon citratus, Tagetus erecta, Chenopodium ambrosioides, Azadirachta indica, Zanthoxylum zanthoxyloides et de Khaya ivorensisaux ont été étudiés. Cependant, contrairement à d'autres ravageurs comme Sitophilus spp, Rhizopertha dominica, Prostephanus truncatus, Tribolium sp ou même Callosobruchus $s p$, peu de travaux sont réalisés sur la lutte contre l'alucite des céréales au moyen d'extraits volatiles de plantes.

Conclusion: Les huiles essentielles des plantes aromatiques du Bénin pourraient être efficaces dans le contrôle des populations de $S$. cerealella dans la conservation des stocks de riz.
\end{abstract}

Mots-clés : Lutte biologique, Sitotroga cerealella, céréales, riz, substances naturelles. 
Status and perspectives of struggle against Sitotroga cerealella (Olivier, 1789), pest of cereals in Benin: a short review

\section{ABSTRACT}

Objective: This review article describes the studies on different methods of fighting against Sitotroga cerealella (Angoumois Grain Moth), and the focus was on the use of non-chemical methods to display a biological fight. $S$. cerealella is one of the most dangerous pests in traditional systems of conservation in Africa and particularly in Benin.

Methods and Results: In the search for information about the struggles against the Angoumois grain moth , several published scientific works were consulted. Examination of the results of research on the control of this pest infesting several cereals revealed frequent use of synthetic chemicals such as deltamethrin, malathion and phosphine fumigation alone or in combination. Several researchers were interested in the physical struggles, ionizing radiation from gamma source of cobalt-60 and resistant varieties of grain in the case of rice, wheat or corn. Also, parasites, pathogens and predators (Trichogramma spp, Blattisocius tarsalis, Cotesia ruficrus, Pteromalus cerealella and Bracon hebetor) were experienced as biological insecticide on different developmental stages of $S$. cerealella. Treatments based on powders, extracts or cooking oils of plants such insecticides like Cymbopogon citratus, Tagetus erecta, Chenopodium ambrosioides, Azadirachta indica, Zanthoxylum zanthoxyloides and Khaya ivorensisaux were studied. However, unlike other pest insects like Sitophilus spp, Rhyzopertha dominica, Prostephanus truncatus, Tribolium sp or even Callosobruchus $s p$, little work is done in the field of fight against Angoumois grain moth.through volatile extracts.

Conclusion: Essential oils from aromatic plants of Benin could effectively control populations of $S$. cerealella in the conservation of stocks of rice.

Keywords: control, Sitotroga cerealella, Cereal, rice, natural substances.

\section{INTRODUCTION}

Les céréales ont de tout temps constitué la principale ressource alimentaire dans le monde. Le stockage, qui est un des moyens sûrs pour répondre aux besoins en semences et pour assurer la sécurité alimentaire, pose un problème auquel une attention toute particulière doit être accordée, si l'on veut limiter les dégâts causés par les déprédateurs des denrées après la récolte. Parmi les insectes ravageurs primaires qui attaquent les céréales en stockage, l'alucite des céréales, Sitotroga cerealella (Olivier, 1789) est considéré comme un insecte dangereux pour les grains stockés et difficile à combattre. En effet, les stades juvéniles de cette espèce (œufs, larves, nymphes et adultes pré-émergents) sont logés à l'intérieur du grain et sont transportés sans possibilité de séparation des grains infestés des grains sains. II est décrit comme l'un des ravageurs primaires les plus redoutables dans les systèmes de conservation traditionnels. Les grains infestés prennent une odeur et un goût désagréables (Kossou et Aho, 1993). Son impact est plus grand dans les régions tropicales et subtropicales, où il attaque le grain dans le champ ainsi que dans le stockage (Seck et al. 1992). L'infestation des stocks de riz par l'alucite des céréales apparaît comme un problème préoccupant dans les zones rizicoles du Bénin. Sa présence est confirmée, et d'importants dégâts peuvent être causés aux stocks de riz dès que la période de stockage s'allonge de trois à quatre mois (Togola et al. 2010). De plus, les échanges de produits céréaliers à travers les frontières, constituent des voies de dissémination possibles du ravageur et les attaques sévères causées par ce ravageur primaire sont de l'ordre de 30 à $85 \%$ dans le cas du riz (Ngamo et al. 2006). Ses attaques se traduisent par la diminution du poids des produits, la baisse du pouvoir germinatif des graines et la perte de leurs valeurs nutritive et marchande. Un facteur important qui contribue à cette grave perte des grains est la tendance des 
larves à nourrir l'intérieur des grains en les évidant. II convient donc de tenir compte de la biologie de l'espèce afin de protéger les récoltes. Les méthodes utilisées pour limiter les pertes dans les stocks sont les moyens physiques qui ont connu de grands succès, la lutte biologiques et l'utilisation des insecticides de synthèse chimique qui sont les plus usitées. Toutefois, ces méthodes présentent plusieurs limites parmi lesquelles l'accoutumance des insectes et la sélection de souches résistantes (Benhalima et al. 2004), les intoxications alimentaires compromettant la santé humaine, la pollution de l'environnement et des désordres écologiques (Regnault-Roger, 2002). Compte tenu des effets néfastes de ces différentes méthodes de lutte en particulier celle de l'utilisation des substances chimiques sur la santé humaine surtout, d'autres alternatives crédibles présentant moins d'impacts négatifs sur l'environnement et la santé humaine méritent d'être explorées (Guèye et al. 2010). Dans cette optique, la valorisation des plantes à effet insecticide semble séduire partout dans le monde. Ainsi, plusieurs organes et parties des plantes sont exploités aux fins de limiter les pertes post-récolte constitue une alternative plus intégrée. Le but de cette revue est de faire l'état des différentes méthodes de lutte développées contre l'alucite des céréales, Sitotroga cerealella et d'envisager quelques perspectives de lutte efficace et respectueuse de la santé humaine et de l'environnement.

\section{LES INSECTES RAVAGEURS DES GRAINS EN POST-RECOLTE}

Le système post-récolte des grains alimentaires est l'ensemble des circuits empruntés par la denrée depuis le champ jusqu'à la table du consommateur (Kossou et Aho 1993). Les pertes les plus importantes sont infligées par différentes espèces de coléoptères, lépidoptères et acariens (Alzouma et al. 1994; FleuratLessard, 1994). Ils sont responsables sous les tropiques de pertes pouvant dépasser $30 \%$. Beaucoup de travaux font référence à l'attaque et à la déperdition des stocks de céréales et de légumineuses par les insectes (Kossou, 1997, Philogène et al. 1989; Ratnadass et al. 1989 ; Ashamo, 2006).

Principaux insectes ennemis des grains stockés en Afrique Occidentale et au Bénin: Les coléoptères constituent de loin le groupe le plus important au sein des insectes ravageurs des stocks. Ils sont suivis par les teignes, les lépidoptères. Ils sont universellement reconnus comme les plus dévastateurs des céréales entreposées en raison de leur propre consommation selon les stades de développement (Markham et al. 1994; Throne, 1994). Les principaux insectes qui vivent dans des stocks au Bénin appartiennent aux ordres des coléoptères et des lépidoptères. Ces insectes nuisibles infestent les denrées et les rendent impropres à la consommation. Au nombre des coléoptères nuisibles aux principales céréales se trouvent Sitophilus zeamais et oryzae, Rhizopertha dominica, Tribolium spp, Oryzaephilus surinamensis, Cryptolestes ferrugineus. Les principaux lépidoptères (teignes) (figure 1) sont Corcyra cephalonica(Pyrale du riz), Sitotroga cerealella(Alucite des céréales), Ephestia cautela,(Pyrale de la farine) et Plodia interpunctella(Pyrale des fruits secs) qui peuvent causer des dégâts appréciables aux grains et farines de maïs, riz, sorgho et mil en stockage. En général, le niveau de population des insectes des stocks est lié à la teneur en eau de la denrée conservée. Le maïs, l'une des principales céréales en Afrique de l'Ouest et particulièrement la plus emblavée au Bénin, est difficile à sécher et donc plus infesté. La sensibilité du maïs aux ravageurs est sans doute la cause du nombre important d'études consacrées à cette céréale. Sitophilus sp, Sitotroga cerealella, Rhyzopertha dominica et surtout Prostephanus truncatus sont les insectes majeurs responsables de la quasi-totalité des dégâts observés sur le maïs. Les dégâts peuvent s'amplifier lorsque l'infestation commence dès le champ (Vowotor et al. 2005). C'est le cas de Prostephanus truncatus et de Sitotroga cerealella sur les stocks de céréales. 


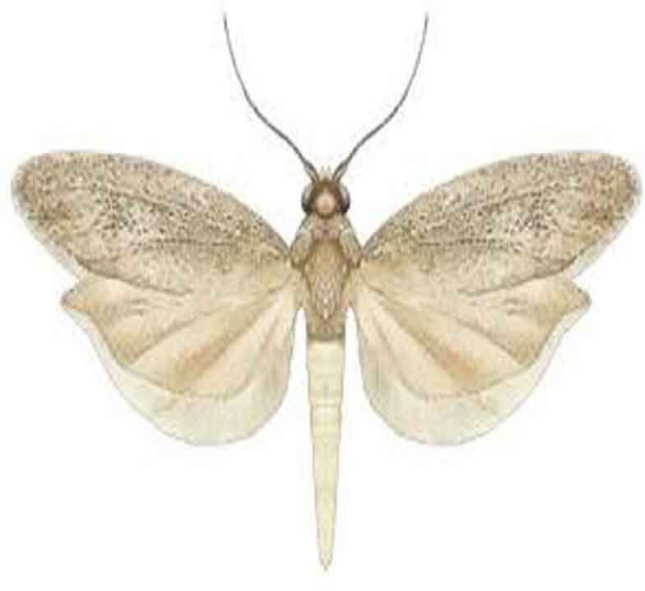

Corcyra cephalonica (Pyrale du riz)

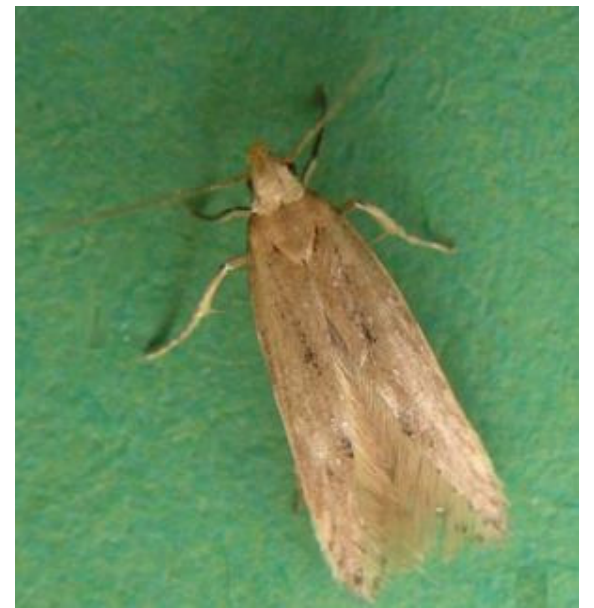

Sitotroga cerealella (Alucite des céréales)

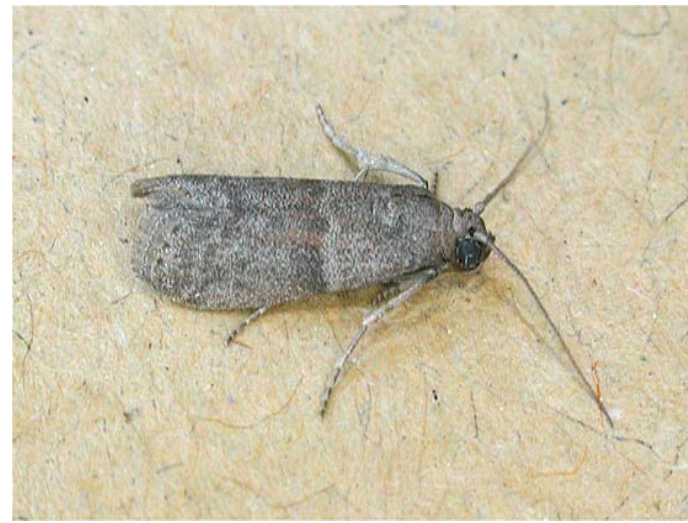

Ephestia cautella (Pyrale de la farine)

Figure 1 : Les principaux lépidoptères des céréales stockées

Cas de Sitotroga cerealella (Olivier 1789), insecte ravageur du riz, émergeant au Bénin : L'alucite des céréales Sitotroga cerealella (Olivier 1789) appartenant à la famille des Gelechiidae et à l'ordre des lépidoptères, est un insecte cosmopolite qui est largement répandu au niveau mondial. C'est est un petit papillon de couleur chamois ou brun jaunâtre qui a une envergure d'environ $1,5 \mathrm{~cm}$. II est le plus fréquemment retrouvé dans les grains infestés et il n'est pas possible de le confondre avec aucun autre. Selon Akter et al. (2013) la longévité des adultes varie entre 2 et 4 semaines selon les conditions de développement. Sitotroga cerealella est un parasite de mais stocké, de blé, de riz, de sorgho et il attaque aussi les céréales dans le champ avant la récolte (Seck, 1991). Sa présence a été signalée sur des stocks paysans de maïs, notamment dans la région des savanes guinéennes au Bénin (Gbati et al. 2002). Cette même étude a indiqué la disparition de l'insecte suite à l'introduction d'ennemis naturels. Nykjaer et al. 2002 ont signalé l'absence de $S$. cerealella au sein de l'entomofaune collectée sur les stocks de maïs dans cette même région. Jusqu'à récemment, l'alucite des céréales, à l'instar d'autres insectes des stocks, ne représentait pas un problème majeur pour le riz au Bénin, car la production rizicole était insuffisante pour 
être conservée pendant plus de trois mois. Actuellement, le riz prend une place importante dans les habitudes alimentaires béninoises, ce qui fait que sa culture s'intensifie et s'étend (MAEP/DFRN, 2000). Aujourd'hui, l'infestation des stocks de riz par l'alucite des grains apparaît comme un problème préoccupant dans les zones rizicoles du pays (Togola et al. 2010). En effet, dans les localités côtières du Sud, l'humidité relative est plus élevée $(80-95 \%)$ et la température oscille entre 20 et $30^{\circ} \mathrm{C}$. Or, le développement des populations de $S$. cerealella est maximal dans cette gamme de températures et d'humidité (Hansen et al. 2002) et les femelles sont plus fertiles (Hansen et al 2004). Les climats humides entraînent aussi une augmentation de la teneur en eau des paddy stockés, créant un environnement favorable à la multiplication de l'alucite (Ratnadass et Sauphanor 1989). Enfin, les régions sud sont également des zones de forts échanges céréaliers avec les pays voisins, ce qui peut accroître la population du ravageur. Pour la zone de Malanville, localité du Nord, cela peut être lié au fait que c'est une zone de grande production de riz irrigué en toute saison, ce qui peut permettre à l'insecte de se multiplier d'autant plus aisément qu'il peut venir des champs (Teetes et al. 1995). En outre, Malanville est une ville frontalière qui reçoit des céréales venant d'autres pays susceptibles d'abriter le ravageur. Aussi une présence plus abondante de $S$. cerealella a été notée sur les stocks de quatre mois (263 alucites par $\mathrm{kg}$ de riz) (Togola et al. 2010). Cet insecte se développe dans les grains, ce qui provoque des dégâts considérables directs (Barney et Weston 1994). Lorsque les conditions sont normales, chaque femelle dépose en moyenne 40 œufs. Les œufs qui sont blancs lorsqu'ils viennent d'être pondus, prennent très tôt la couleur rougeâtre. A l'éclosion, la jeune larve se glisse vers l'albumen du grain et elle tisse souvent un petit cocon d'entrée. Après sa pénétration, elle se nourrit soit de l'endosperme, soit du germe jusqu'à son complet développement. En ce moment elle creuse une galerie vers l'extérieur de la semence et prépare un trou de sortie en découpant la coque sur la moitié ou les trois-quarts de sa circonférence, formant un opercule faiblement fixé. Plus tard l'adulte sort par l'opercule partiellement découpé (Raoelijaona, 1996). Dans des conditions de forte infestation, les produits stockés peuvent subir $100 \%$ de pertes. Les attaques de $S$. cerealella se traduisent par la diminution du poids des produits, la baisse du pouvoir germinatif des graines et par la perte de la valeur nutritive et de la valeur marchande.

\section{MOYENS DE LUTTE CONTRE L'ALUCITE DES CEREALES}

De nombreuses expériences et applications ont été réalisées avec divers produits chimiques de synthèse et autres formes de luttes dans le contrôle des alucites des céréales. II s'agit des luttes physique, biologique ou de plus en plus l'utilisation de poudres et des huiles essentielles qui sont des alternatives à la lutte chimique.

La lutte chimique: L'examen des résultats de recherches réalisées montre que les traitements insecticides et la fumigation standard sont généralement efficaces contre $S$. cerealella. Phoxim et pirimiphos-méthyle (2-diéthylamino-6-méthyl pyrimidin4-yl dimethyl phosphorothionate) ont été trouvées efficaces pour assurer une protection contre $S$. cerealella sur du riz en sac pour un maximum de 6 mois au cours des essais en Guyane (Rai et Croal 1973). Les recherches en Illinois de 1959 à 1960 sur le malathion ont révélé d'une part que l'un de ses composants, le xanthoxylin a eu des effets disuasifs importants sur le comportement de ponte et a eu de fortes propriétés répulsives sur les larves de $S$. cerealella et d'autre part, les poudres et jets du malathion ont d'excellents contrôles sur le ravageur avec une réduction moyenne de $89,4 \%$ en dommages obtenue pour tous les traitements durant les deux saisons de tests. Grâce à ce traitement, l'effectif d'autres insectes nuisibles des stocks de céréales a été réduit de $80 \%$. Le méthacrifos a été comparé avec le malathion pour la protection du maïs stocké en paille contre S. cerealella et Sitophilus zeamais au Brésil. Les meilleurs résultats ont été obtenus avec une combinaison de fumigation (avec de la phosphine) et le traitement avec le méthacrifos. L'utilisation de la phosphine mélangée avec du dioxyde de carbone $\left(\mathrm{CO}_{2}\right)$ contre les populations d'alucite des grains, a produit une mortalité significativement plus élevée des larves (El-Lakwah et al. 1991). Dans d'autres études sur l'utilisation de la décaméthrine contre $S$. cerealella et $S$. zeamais, la fumigation suivie d'un traitement avec de la deltaméthrine a donné la meilleure protection. De même, le fenvalérate et le malathion ont été trouvés efficaces contre $S$. cerealella sur le riz en Inde (Dakshinamurthy et Regupathay 1992). La deltaméthrine et la perméthrine quant à elles, sont 
également signalées pour donner de bons niveaux de protection contre un certain nombre de ravageurs du grain entreposé, y compris $S$. cerealella (Hung et al 1990). Aussi la protection par la deltaméthrine des grains de mil infestés artificiellement avec des adultes de Sitotroga cerealella, s'est révélée plus efficace lors des tests. En effet, elle assure un contrôle total de 1'émergence de la F1, même avec la dose de 0,625 ppm, et fait preuve, par rapport aux deux organophosphorés à savoir fénitrothion et pyrimiphosméthyl, d'une plus grande toxicité résiduelle et d'une plus grande persistance de la matière active. Les pulvérisations répétées de la poussière et tetrachlorvinphos carbaryl ont été utilisées pour contrôler S. cerealella au Bangladesh (Bhuiyah et al. 1992), en outre le fenoxycarb empêche la reproduction de $S$. cerealella. Dans la région administrative de Sidamo en Ethiopie après le dépistage de plusieurs insecticides pour le contrôle de $S$. cerealella, le primiphos méthyle, la deltaméthrine et le primiphosmythyl + perméthrine et malathin + perméthrine ont montré un contrôle complet de l'organisme nuisible (Emana, 1993). Un certain nombre de produits naturels ont également été utilisés pour contrôler $S$. cerealella. II s'agit notamment de biogaz provenant du fumier de bovins (Jin et Pan 1983), constitué de $60 \%$ de méthane, $30-35 \%$ de dioxyde de carbone et des traces d'autres gaz (Palaniswamy et Dakshinamurthy 1986).

Résistance de l'alucite des céréales aux insecticides: La résistance des insectes aux pesticides de synthèse est l'un des principaux méfaits de l'application répétée des produits contre les ravageurs. S. cerealella semble avoir développé une certaine résistance au malathion et au phoxim à Taiwan (Kao et Tzeng 1992). Seck (1992b) a constaté que le fénitrothion, le fenthion et le pirimiphos-méthyl ne donnent pas un contrôle satisfaisant vis-à-vis de $S$. cerealella au Zimbabwe. L'ensemble des cas évoqués ci-dessus montre la nécessité d'associer la lutte chimique à d'autres techniques qui seront à même de confiner les dégâts des insectes dans des limites économiquement supportables, tout en assurant un environnement moins exposé aux pollutions chimiques. Actuellement, aucun groupe parmi les organophosphorés, organochlorés, pyréthrinoïdes ou encore fumigants n'échappe à la résistance des insectes.

Les alternatives à la lutte chimique: En effet, le choix de l'insecticide appliqué dépend de la durée de l'entreposage et de la sécurité relative du composé pour les applicateurs, les consommateurs et l'environnement. Face donc aux nuisances de la lutte chimique et les cas de résistances aux produits chimiques de synthèse nonobstant les succès enregistrés, il a été développé plusieurs autres formes de lutte contre les insectes et aussi vis-à-vis de Sitotroga cerealella.

La résistance variétale: Des variétés plus tolérantes aux insectes ont été développées dans le but de limiter les pertes. La résistance des génotypes des grains de douze variétés de blé prometteurs a été évaluée pour la résistance à la pyrale du grain, Sitotroga cerealella fondée sur la descendance de papillons émergés et la perte de poids des grains a révélé que la population de pyrale était significativement plus faible avec la perte de poids de grain minimum avec Chakwal-86 et Bleu Argent suivie par le Pasban-90 et du Parwaz-94 qui indique la résistance à $S$. cerealella. (Shafique, 2006a). L'évaluation de la résistance relative des variétés de riz à l'attaque par Sitotroga cerealella a révélé que les variétés "indonésiennes» étaient relativement résistantes aux attaques de $S$. cerealella par rapport aux variétés "françaises», sauf la variété Delta sous la forme de paddy (Rizwana et al. 2011).

Lutte physique: Elle concerne toutes les techniques mécano-thérapeutiques susceptibles de rendre le stock sain. En général, ces techniques ne sont pas efficaces contre les formes cachées. Elles sont recommandées pour pallier aux problèmes des résidus chimiques liés aux différents traitements chimiques appliqués aux denrées stockées. Plusieurs techniques ont été expérimentées et certaines ont eu des succès divers: l'écrasement mécanique dans les "Entôler ", le stockage étanche ou sous atmosphère contrôlée et les irradiations ionisantes. Cette dernière méthode a focalisé l'attention de plusieurs chercheurs dans la lutte physique contre $S$. cerealella. Avec l'irradiation gamma entre 75 et $175 \mathrm{~Gy}$, le pourcentage de stérilité des pulpes de l'alucite des grains, Sitotroga cerealella a augmenté avec l'augmentation de la «dose d'irradiation gamma ». En outre, le pourcentage de la stérilité a augmenté en $F_{1}$ et a provoqué une diminution de la taille. En utilisant des doses allant de 336 à $388 \mathrm{~Gy}$ on a pu empêcher l'éclosion des œufs de la $F_{1}$ sur un total de 22083 papillons indiens. Dans cette même optique, il a fallu des gammes de doses de 443 à 505 et 590 à $674 \mathrm{~Gy}$, respectivement, pour empêcher l'éclosion des œufs de la $F_{1}$ sur un total de 15264 et 13677 adultes de Sitotroga cerealella irradiés dans des atmosphères ambiantes et hypoxiques (Vincent et al. 2003 ; Hallman et al. 2008). Des doses létales d'irradiation provenant d'une source de rayons gamma du cobalt 60 sur la 

1789) de prédateur des céréales au Benin

fertilité des adultes de $S$. cerealella ont provoqué la chute à $2 \%$ la fécondité des femelles lorsque ces dernières ont été irradiées avec une dose de $70 \mathrm{Krad}$. Par ailleurs la sensibilité des différents stades de Sitotroga cerealella aux radiations gamma a révélé que les progénitures, sous l'effet de ces radiations, à partir des parents irradiés, a engendré des anomalies dans la structure des spermatozoïdes. Les mêmes études sont réalisées sur la fécondité, la longévité et la stérilité des adultes de Sitotroga cerealella. Une réduction de la fécondité des femelles suivant l'augmentation de la dose de 10 à 90 Krad a été notée. Par rapport aux témoins, la longévité est réduite chez les insectes exposés à $100 \mathrm{Krad}$. L'accouplement entre des mâles et des femelles également traités par $20 \mathrm{Krad}$ a provoqué $97.17 \%$ de stérilité chez les femelles (Ismail et al. 1987). Quant aux contrôles des œufs et des adultes de 0 à 24 heures, irradiés avec des doses de 0 , $1,2,4,6,8,10$ et $12 \mathrm{Krad}$, il a été observé que $10 \mathrm{Krad}$ ont tué tous les embryons. En outre, lorsque soit les mâles ou les femelles avaient reçu la dose de 100 $\mathrm{Krad}$, tous les insectes sont morts au bout de deux jours. (Rodrigues et al. 1983). L'irradiation connaît beaucoup de succès ces dernières années. Ses principaux avantages sont l'absence de résidus et de résistance, sa capacité de pénétration dans des denrées même emballées et la rapidité de son application. Aux doses prescrites, l'irradiation n'altère aucune des propriétés physiques, chimiques et organoleptiques de la denrée. Quant à l'atmosphère contrôlée, les infrastructures de stockage sont enrichies en azote ou en dioxyde de carbone dans le but de réduire la teneur en oxygène et d'asphyxier les insectes. D'une manière générale, cette technique s'avère inapplicable en milieu paysan compte tenu de l'équipement et des connaissances nécessaires pour sa mise en œuvre (Seck, 2009). II s'avère donc indispensable de développer d'autres techniques de lutte adaptées au contexte socio-économique des populations rurales.

Lutte biologique: Généralement ce mode de lutte s'articule dans la majeure partie des cas sur l'utilisation de parasitoïdes, parasites et prédateurs. Dans le cas du maïs, une expérimentation conduite au Bénin en laboratoire et dans les champs a révélé les possibilités d'utilisation d'isolat de conidies de Beauveria bassina contre P. truncatus (Meikle et al. 2001). S'agissant du ravageur Sitotroga cerealella, le recours aux ennemis naturels a été effectué dans le contrôle de différents stades de son développement (Haines, 1998 ; Schöller, 1998). Les meilleurs résultats sont obtenus avec les parasites Pteromalus cerealellae et Bracon hebetor sur les larves de Sitotroga cerealella dans l'ex-URSS (Astanov, 1980) et en Arabie saoudite (Mostafa et al. 1981). Les Trichogramma spp. ont été signalés comme des parasites des œufs de $S$. cerealella dans l'exURSS (Zil'berg et Filipchuk 1976) et en Pologne (Olszak et Bakowski 1976). Blattisocius tarsalis a été également signalé comme un acarien prédateur des œufs de S. cerealella. L'insecte Cotesia ruficrus, parasite du lépidoptère Mythimna separata, peut également avoir un potentiel comme agent de lutte biologique contre S. cerealella (Mundiwale et al. 1984), mais n'a pas encore été enregistré dans le domaine. Malgré les chances de succès, le constat est que la lutte biologique tarde à prendre son essor dans beaucoup de pays ouest-africains. Certains facteurs concourent à limiter l'emploi de techniques assez exigeantes voire sophistiquées dans les zones rurales africaines particulièrement concernées par les pertes post-récolte. Ekesi et al. (2001) soulignent que l'efficacité des entomopathogènes peut être affectée par de fortes intensités lumineuses et les rayons ultraviolets. Ainsi, un stockage de longue durée dans de telles localités s'avère difficile, surtout si l'on sait que beaucoup de localités sont dépourvues d'énergie et d'installations adéquates pour la conservation de tels organismes. Ces limites que sont surtout le retard pour son essor, l'exigence ou le caractère sophistiqué de ces techniques et de nature à nous inciter à rechercher d'autres moyens utilisant des techniques moins coûteuses et dont la mise en œuvre par les producteurs serait moins contraignante.

\section{UTILISATION DES PLANTES INSECTICIDES DANS LE CONTROLE DE S. CEREALELLA}

Toxicité des poudres et les extraits des plantes insecticides : L'usage des plantes indigènes dans la conservation des récoltes a été pratiqué avant même l'apparition des insecticides de synthèse. Les plantes sont utilisées contre les ravageurs pour leurs effets répulsifs, de contact ou fumigant. Boeke et al. (2004) ont relevé une action répulsive et toxique de Tephrosia vogelii (Hook f.) sur C. maculatus, alors que Blumea aurita (L.), qui ne présente aucune toxicité, possède un fort pouvoir répulsif sur le même insecte. Les extraits de Acorus calamus (L.), Azadirachta indica (A. Juss) et Curcuma longa (L.) préparés dans l'éther de pétrole, 
l'acétone et l'éthanol ont été évalués comme inhibiteur de croissance contre Sitotroga cerealella Parmi ces extraits, l'extrait de l'éther de pétrole de $A$. calamus aux taux d'application de 1000, 500 et $250 \mathrm{pg} / \mathrm{g}$ et son extrait d'acétone à 1000 et $500 \mathrm{pg} / \mathrm{g}$ ont complètement inhibée l'émergence des adultes (Javed et al. 2010). Les feuilles séchées de sauge sauvage Lippia geminata ont également été identifiées comme un répulsif efficace contre $S$. cerealella pour le riz stocké en Inde pour une durée de 9 mois maximum (Prakash et Rao, 1984). Les extraits de neem ont été plus toxiques dans le contrôle de la teigne du riz sur grains de riz stocké variété BR-11 infestée (Akter et al. 2013). Au cours de ses essais Fekadu (2013) a montré que les poudres et les huiles de cuissons d'écorce d'Azadirachta indica et des feuilles de Cymbopogon citratus ont provoqué $77,8 \%$ de mortalité de Sitotroga cerealella contre $94 \%$ pour le contrôle standard avec le malathion. Les poudres et extraits des plantes de Aristolochia ringens L., Khaya ivorensis (K.), Strophanthus hispidus (DC) et Zanthoxylum zanthoxyloides Lam. testés contre $S$. cerealella étaient capables d'effectuer $100 \%$ de mortalité des papillons adultes dans les $24 \mathrm{~h}$ d'application pour toutes les concentrations (Ashamo et Akinnawonu 2012). Aussi, les extraits aqueux de Nerium lauriers et Azadirachta indica ont-ils réduit complètement l'infestation des larves de S. cerealella et induit une stérilité de $50 \%$ chez les adultes émergents du maïs. Les autres extraits, Graveolens Anethum et Lantana camara ont réussi à limiter les dégâts et la viabilité des larves.

Les huiles essentielles: Les plantes ont été longtemps utilisées par les paysans pour exhauster le goût des aliments ou pour protéger les produits récoltés (Keita et al. 2000; Isman, 2000). Les huiles essentielles extraites de ces plantes ont été largement utilisées dans la lutte contre les ravageurs de stocks (Kossou, 1989; Regnault-Roger et Hamraoui 1993 ; Liu et Ho 1999; Tunç et al. 2000 ; Ketoh et al. 2005; Noudogbessi et al. 2008 et 2009). Leurs propriétés insecticides, larvicides, ovicides, stérilisantes, antiappétentes, répulsives ont fait l'objet de nombreuses études (Regnault-Roger et Hamraoui 1997 ; Teugwa et al. 2002). La sensibilité d'un insecte vis-à-vis d'une huile essentielle évolue en fonction de son cycle biologique. En effet, les récepteurs chimiques chez les insectes n'existent pas dans le système nerveux des mammifères. A cause de leur faible persistance, les huiles essentielles des plantes aromatiques ne présentent pas de risques pour l'environnement. Leur activité peut varier en fonction des stades du cycle de vie d'un insecte (RegnaultRoger, 2002). Les composés chimiques responsables des actions sur les ravageurs de stock sont principalement les monoterpènes (1-8 cinéole, l'eugenol, le camphor) à effet insecticide sur la survie de C. maculatus ou S. zeamais (Kouninki et al. 2007 ; Noudogbessi et al. 2009) ou des alcaloïdes à effet inhibiteur du développement larvaire des ravageurs comme T. castananeum (Ngamo et al. 2001). Les recherches menées dans le but d'étudier l'effet insecticide, ovicide et larvicide de certaines plantes aromatiques sur Sitotroga cerealella, un des ravageurs les plus redoutables enregistrées des céréales dans le monde entier sont, à ce jour, peu nombreuses dans la littérature. Les activités de fumigation, d'influence du comportement et de l'inhibition sur $S$. cerealella ont été évaluées à partir de trois matériaux, l'huile essentielle de l'ail (Allium sativum) ou garlic et deux de ses composantes, le disulfure de diallyle et trisulfure de diallyle. Ils ont agi comme fumigènes, produits de dissuasion comportementale dans l'olfactomètre $Y$-tube et comme inhibiteurs de ponte contre l'alucite des grains (Feng-Lian, 2011). La toxicité par fumigation de l'huile essentielle de semences de neem aux doses de $25-200$ pi a causé $100 \%$ de mortalité des adultes et des larves. La toxicité des extraits de Eugenia aromatica (L) dans la protection de six variétés à haut rendement de riz paddy NERICA infestées par $S$. cerealella ont montré que l'extrait produit une faible émergence des adultes alors que la poudre avait moins d'activité insecticide produisant plus d'émergence des adultes avec toutes les gammes de concentrations utilisées, mais mieux que le contrôle. Aussi le traitement n'a pas affecté la viabilité et la capacité d'absorption d'eau des grains par rapport au traitement contrôle (Aringbangba, 2011). De nos jours, la lutte contre les ravageurs des produits agricoles entre dans une nouvelle phase avec le regain d'intérêt pour les biopesticides d'origine végétale. Les recherches de nouvelles molécules biologiquement actives des populations contre Sitotroga cerealella doivent se poursuivre en tenant compte du critère efficacité tant en laboratoire que sur le terrain. 


\section{PERSPECTIVES DE CONTROLE DE SITOTROGA CEREALELLA AU BENIN}

Les plantes sont localement disponibles, moins chères et plus faciles d'emploi qu'un insecticide de synthèse. Les méthodes traditionnelles de lutte ainsi que les pratiques d'avant stockage réduisent de manière significative les infestations par les insectes. L'utilisation des insecticides naturels doit être encouragée par plus de recherche de terrain et d'aide au développement, de technologie pour l'extraction ou la transformation des composés naturels. Les différentes méthodes présentées comme alternatives aux pesticides présentent chacune des avantages, mais aussi quelques limites. C'est là tout l'intérêt d'une gestion intégrée basée sur la combinaison de plusieurs procédés pour circonscrire l'activité des insectes, redoutables compétiteurs de l'homme. Les différences de sensibilité des insectes aux produits devraient nous amener à considérer la lutte de manière plus spécifique. En effet, le choix de l'insecticide (naturel) devra en priorité reposer sur l'insecte majeur à combattre. II a été prouvé que la réponse à l'intensité de la toxicité d'un produit dépendait dans bien des cas de manière spécifique du ravageur et pour un ravageur donné du stade considéré. L'efficacité des plantes n'est pas non plus toujours garantie avec tous ses organes et dépend du stade phénologique. De plus, l'efficacité des biocides est en partie associée aux conditions

\section{REFERENCES BIBLIOGRAPHIQUES}

Akter T, Jahan M. 2013. Toxicité Effet de la plante indigène couramment utilisés des extraits Contrôle de la teigne du riz, Sitotroga cerealella Oliv. En stockée grain de riz. International Journal of Sustainable Agriculture 5 (1): 10-15

Alzouma L, Huignard L, Lenga A. 1994. Les coléoptères Bruchidae et les autres insectes ravageurs des légumineuses alimentaires en zone tropicale. In Post-Récolte, principes et application en zone tropicale, ESTEM/AUPELF, p.79-103.

Aringbangba Rufus Oluwatuyi. 2011. Toxicity of extract from Eugenia aromatica (I.) on sitotroga cerealella (olivier) (lepidoptera: gelechiidae) infesting different varieties of paddy rice. A thesis submitted to the school of post graduate studies in partial fulfilment of the requirements for the award of master of technology degree in food storage technology. Department of pédoclimatiques. L'objectif d'autosuffisance alimentaire défini dans la plupart des pays africains, dépend largement des ravageurs des grains et graines impliqués. Mais cette approche, au risque de ne pas être applicable, ne doit jamais perdre de vue le contexte et la réalité de l'agriculture africaine, qui est une agriculture essentiellement de type paysan. L'objectif à atteindre est donc de mettre au point des méthodes de stockage efficaces, simples, sûres et surtout accessibles aux agriculteurs. Pour y parvenir et compte tenu des succès et des limites des résultats qui précèdent, les principaux axes de recherches à court et moyen termes pourraient être orientés surtout sur l'approfondissement des études sur les plantes aromatiques dotées de pouvoir réel insecticide, ovicide, larvicide, anti appétant, répulsif ou dissuasif sur les alucites des grains, Sitotroga cerealella, déprédateur des céréales. En effet, peu d'études ont été effectuées dans ce domaine et cette perspective fournira des informations utiles dans le contrôle efficace contre ce ravageur en Afrique et particulièrement au Bénin. La flore béninoise est très riche et diversifiée. Beaucoup d'autres plantes à essences restent encore à découvrir et dont les huiles essentielles peuvent se révéler très efficaces contre Sitotroga cerealella, ennemi premier des stocks de riz et d'autres céréales du Bénin.

Biology, Federal University of Technology, Akure. Pages 91.

Ashamo MO, Akinnawonu O. 2012. Insecticidal efficacy of some plant powders and extracts against the Angoumois grain moth, Sitotroga cerealella (Olivier) (Lepidoptera: Gelechiidae) Archives Of Phytopathology And Plant Protection Volume 45, Issue 9, pages 1051-1058.

Ashamo MO. 2006. Relative susceptibility of some local and elite rice varieties to the rice weevil, Sitophilus oryzae L. (Coleoptera: Curculionidae). J. Food Agric. Environ., 4(1), 249-252.

Astanov T. 1980. A kolkhoz laboratory rears insect enemies. Zashchita Rastenii, No. 11:13

Barney RJ, Weston PA. 1994. Grain storage in a smallfarm ecosystem: Angoumois grain moth movement and management. Proc. 6th Int. Work. Conf. Stored-product Prot., Canberra, 1:383-384. 
Benhalima H, Chaudhry MQ, Mills KA, Price NR. 2004. Phosphine resistance in stored-product insects collected from various grain storage facilities in Morocco. J. Stored Prod. Res., 40, 241-249.

Bhuiyah MIM, Alam S, Karim ANMR. 1992. Losses to stored rice caused by rice weevil and angoumois grain moth, and their control in Bangladesh. Bangladesh Journal of Agricultural Sciences, 19(1):13-18

Boeke SJ, Baumgart IR, van Loon JJA, van Huis A, Dicke M, Kossou DK. 2004. Toxicity and repellence of African plants traditionally used for the protection of stored cowpea against Callosobruchus maculatus, Journal of Stored Products Research, 40: 423-438.

Dakshinamurthy A, Regupathy A. 1992. Pre-harvest spray of insecticides for the management of Sitotroga cerealella Olivier on rice. Bioecology and control of insect pests: Proceedings of the National Symposium on Growth, Development \& Control Technology of Insect Pests [edited by Goel, S. C.] Muzaffarnagar, India; Uttar Pradesh Zoological Society, 218-224

Ekesi S, Egwurube EA, Akpa AD, Onu I. 2001. Laboratory evaluation of the entomopathogenic fungus, Metarhizium anisopliae for the control of the groundnut bruchid, Caryedon serratus on groundnut. J. Stored Prod. Res., 37, 313-321.

El-Lakwah F, Meuser F, Abdel Gawad A, Wohlgemuth R, Darwish A. 1991. Efficiency of phosphine alone and in mixtures with carbon dioxide against Angoumois grain moth Sitotroga cerealella (Olivier), (Gelechiidae, Lepidoptera). Zeitschrift fuer Pflanzenkrankheiten und Pflanzenschutz (Germany, F.R.)

Emana G. 1993. Studies on the distribution and control of Angoumois grain moth (Sitotroga cerealella) in Sidamo Administrative Region. M.Sc. Thesis, Alemaya University of Agriculture, Alemaya

Fekadu Gemechu, Waktole Sori, Dante R. Santiago. 2013. Efficacy of botanical powders and cooking oils against Angoumois grain moth, Sitotroga cereallela O. (Lepidoptera: Gelechiidae) in stored maize. African Journal of Biotechnology. Vol. 12(16), pp. 1978-1986.

Feng-Lian Y, Fen ZCL. 2011. Insecticidal activities of garlic substances against adults of grain moth, Sitotroga cerealella (Lepidoptera: Gelechiidae)
German National Library of Science and Technology (Getlnfo) TIBscholar

Fleurat-Lessard F. 1994. Écophysiologie des Arthropodes nuisibles aux stocks de céréales en Afrique tropicale. In Post-Récolte, principes et application en zone tropicale, ESTEMIAUPELF Verstraeten, 1-61.

Gbati O, Hell K, Azoma, Skovgaard H. 2002. Danish Pest Infestation Laboratory Annual Report (Danemark).

Guèye MT, Seck D, Wathelet JP, Lognay G. 2010. Lutte contre les ravageurs des stocks de céréales et de légumineuses au Sénégal et en Afrique occidentale : synthèse bibliographique . Biotechnol. Agron. Soc. Environ. 2011 15(1), 183-194

Haines CP. 1998. Arthropod natural enemies in stored product - overlooked and under-exploited. Proc. 7th Int. Work. Conf. Stored-product Prot., Beijing, 2:1205-1226.

Hallman GJ, Phillips TW. 2008. Ionizing Irradiation of Adults of Angoumois Grain Moth (Lepidoptera: Gelechiidae) and Indianmeal Moth (Lepidoptera: Pyralidae) to Prevent Reproduction, and Implications for a Generic Irradiation Treatment for Insects Journal of Economic Entomology 101(4):1051-1056.

Hansen LS, Skovgaard H. 2002. Development time and age-specific fecundity of Sitotroga cerealella in relation to temperature and relative humidity. Annual Report. Lyngby (Danemark): Danish Pest Infestation Laboratory.

Hansen LS, Skovgard S, Hell K. 2004. Life Table Study of Sitotroga cerealella (Lepidoptera: Gelichiidae), a Strain from West Africa. Journal of Economic Entomology; 97: 1484-90.

Hung CC, Hsieh FK, Hwang JS. 1990. Stored grain insects monitoring and their chemical control. Chinese Journal of Entomology, 10(2):169-179

Ismail II, El-Nahal KM, Kamel AH, Mostafa TS. 1987. Effect of sublethal doses of gamma radiation on the fecundity, longevity and sterility of adults of Sitotroga cerealella (Olivier) (Lepidoptera, Gelechiidae). Insect science and its application.

Isman MB. 2000. Plant essential oils for pest and disease management. Crop Protection. 19: 603-608.

Javed Iqbal, Ghulam Jilani, Muhammad Aslam. 2010. Growth Inhibiting Effects of Plant Extracts Against the Grain Moth, Sitotroga cerealella 
(Oliv.) (Gelechiidae: Lepidoptera) Pakistan J. Zool., vol. 42(5), pp. 597-601

Kao SS, Tzeng CC. 1992. A survey of the susceptibility of rice moth (Corcyra cephalonica) and Angoumois grain moth (Sitotroga cerealella) to malathion and phoxim. Chinese Journal of Entomology, 12(4):239-245

Keita SM. 2000. Effect of various essential oils on Callosobruchus maculatus (F.) (Coleoptera:Bruchidae). J. Stered Prod. Res., 36,355-364p.

Ketoh GK, Koumaglo HK, Glitho IA. 2005. Inhibition of Callosobruchus maculatus (F.) (Coleoptera: Bruchidae) development with essential oil extracted from Cymbopogon schoenanthus $\mathrm{L}$. Spreng. (Poaceae), and the wasp Dinarmus basalis Rondani (Hymenoptera: Pteromalidae). J. Stored Prod. Res., 41, 363-371.

Kossou KD. 1997. Les insecticides naturels: Une composante non négligeable dans la lutte intégrée contre les ravageurs post-récolte, Actes du Premier Colloque International du Réseau Africain de Recherche sur les Bruches, Lomé, 139.

Kossou KD, Aho N. 1993. Stockage et conservation des grains alimentaires tropicaux. Principes et pratiques. Flamboyant Ed. Cotonou, $125 \mathrm{p}$.

Kossou KD. 1989. Evaluation des produits du neem Azadirachta indica A. Juss pour le contrôle de Sitophilus zeamais Motsch sur le maïs en postrécolte. Insect Sci. Applic. 10 (3):365-372.

Kouninki H, Hance T, Noudjou FA, Lognay G, Malaisse $F$, Ngassoum MB, Mapongmetsem PM, Ngamo LST, Haubruge E. 2007. Toxicity of some terpenoids of essential oils of Xylopia aethiopica from Cameroon against Sitophilus zeamais Motchulsky. Journal of Applied Entomology, document online, 8p.

Liu ZL, Ho SH. 1999. Bioactivity of the essential oil extracted from Evodia rutaecarpa Hook f. et Thomas against the grain storage insects, Sitophilus zeamais Motsch. and Tribolium castaneum (Herbst). J. Stored Prod. Res., 35, 317-328.

MAEP/DFRN. 2000. Base des données géoréférencées sur l'utilisation agricole des terres au Bénin. Rapport final. Cotonou : CENATEL.

Markham, RH, Bosque-Pérez NA, Borgemeister C, Meikle WG. 1994. Developing pest management strategies for Sitophilus zeamais and Prostephanus truncatus Tropics FAO plan prot. 42: 97-116.

Meikle WG, Holst N, Markham RH. 2001. The effects of an entomopathogenic fungus, Beauveria bassiana (Balsamo) Vuillemin (Hyphomycetes), on Prostephanus truncatus (Horn) (Col.: Bostrichidae), Sitophilus zeamais Motschulsky (Col.: Curculionidae), and grain losses in stored maize in the Benin republic. J. Invertebr. Pathol., 77, 198-205.

Mostafa SAS, Dabbour Al, Nassif MA, Aziz MIA. 1981. Insects damaging stored products in Saudi Arabia. Anzeiger fur Schadlingskunde Pflanzenschutz Umweltschutz, 54(12):184-187

Mundiwale SK, Men UB, Borle MN. 1984. A record of new parasite on armyworm. Indian Journal of Entomology, 46(2):247-248

Ngamo LST, Hance Th. 2006. Diversité des ravageurs des denrées et méthodes alternatives de lutte en milieu tropical. 25(4), 215-220.

Ngamo LST. 2001. Deuxième rapport annuel 20002001. Grand programme de recherche Universitaire. Développement de la valorisation des ressources animales et végétales. Protection intégrée des ressources stockées Université de N'Gaoundéré. 15p.

Noudogbessi J-P, Kèkè $M$, Avlessi $F$, Kossou D, Sohounhloué DCK. 2009. Evaluation of the insecticidal, larvicidal and ovicidal effects on Callosobruchus maculatus of essential oils of Cymbopogon giganteus and of Xylopia aethiopica. SCIENTIFIC STUDY \& RESEARCH $\vee$ Vol. $(X)$ 4, 337-350x.

Noudogbessi J-P, Kossou D, Sohounhloué DCK. 2008. Effet Insecticide, Ovicide et Larvicide des Huiles Essentielles de Pimenta racemosa (Miller) et de Chromolaena odorata (L. Robinson) sur le Grand Capucin (Prostephanus truncatus (Horn)) du Maïs. J. Soc. Ouest-Afr. Chim. 026; $41-51$.

Nykjær M, Skovgard H. 2002. Intra- and interspecific competition between Sitophilus zeamais and Sitotroga cerealella. Lyngby (Danemark) : Danish Pest Infestation Laboratory. Annual Report (Danemark).

Olszak R, Bakowski G. 1976. Mass rearing of the Angoumois grain moth - Sitotroga cerealella Oliv. (Lepidoptera, Gelechiidae). Polskie Pismo Entomologiczne, 46(1):187-200 
Palaniswamy PT, Dakshinamurthy A. 1986. Biogas to control rice storage pests. International Rice Research Newsletter, 11(6):25

Philogène BJR, Armson JT, Lambert JDH. 1989. Facteurs contribuant à la protection du maïs contre les attaques de Sitophilus et Prostephanus. In: Parmentier M. \& Kouahou F.-B., eds. Céréales en régions chaudes. Montréal, Canada: Aupelf-Uref; Paris : John Libbey Eurotext, 47-56.

Prakash A, Rao J. 1984. Wild sage, Lippia geminata: a paddy grain protectant in storage. Oryza, 21(4):209-212

Rai BK, Croal K. 1973. Chemical control of Sitotroga cerealella (Olivier) infesting paddy stored in bags. Indian Journal of Entomology, 35(1):5762

Raoelijaona C. 1996. Les insectes ravageurs du riz, du maïs stockés et des légumineuses sèches. Recueil des exposés lors du symposium à Toliara du 7 au 11 octobre 1996. Antananarivo : Service de la surveillance phytosanitaire du territoire national.

Ratnadass A, Sauphanor B. 1989. Les pertes dues aux insectes sur les stocks paysans de céréales en Côte d'Ivoire. In: Parmentier M. \& Kouahou F.-B., eds. Céréales en régions chaudes. Montréal, Canada: Aupelf-Uref ; Paris : John Libbey Eurotext, 141-150.

Regnault-Roger C, Hamraoui A. 1997. Lutte contre les insectes phytophages par les plantes aromatiques et leurs molécules allelochimiques. Acta Bot. Gallica, 144, 401412.

Regnault-Roger C. 2002. De nouveaux phytoinsecticides pour le troisième millénaire. In: Philogène BJR, Ragnault-Roger C, Vincent C. coord. Biopesticide d'origine végétale. Paris: Lavoisier-Editions Tec et Doc, 19-39p

Rizwana S, Naheed MHA, Afghan S. 2011. Resistance in Stored Rice Varieties Against Angoumois Grain Moth, Sitotroga cerealella (Olivier) (Lepidoptera: Gelechiidae) Pakistan J. Zool., vol. 43(2), pp. 343-348.

Rodrigues ZA, Rego AM, Oliveira ML, Ferreira D. 1983. Effects of gamma radiation from Cobalt 60 on eggs and adults of Sitotroga cerealella (Oliver, 1819) (Lepidoptera, Gelechiidae) in laboratory. Pernambuco Univ., Recife (Brazil). Dept. de Energia Nuclear).
Schöller M. 1998. Biological control of arthropod pests in stored product protection with predators and parasitoids - Review and bibliography. Mitt. Biol. Bundesanst. Land-Forstwirtsch., 342:85189.

Seck D. 2009. Stockage, conservation et transformation des récoltes en zones tropicales. Notes de cours, 31P.

Seck D, Sidibé B, Fall A. 1992. Observations sur le développement de Tribolium castaneum Herbst sur mil (Pennisetum thyphoïdes L.), en fonction du taux de brisure dans le substrat. Mém. Soc. R. Belge Entomol., 35, 471-475.

Seck D. 1992b. Evaluation de l'efficacité biologique et de l'action résiduelle de troismatières actives sur Sitotroga cerealella (Oliv.) (Lep. Gelechiidae), ravageur du milstocké. Mem. Soc. R. Ent. Belge (sous presse).

Seck D. 1991. Étude de l'infestation initiale de Sitotroga cerealella (Oliv.) en fonctionde la localisation des champs de mil (Pennisetum typhoïdes). Insect Sci. Applic. Vol. 12, N" 5/6 pp. 507509.

Shafique M, Ahmad M, Chaudry MA. 2006a. Evaluation of Wheat Varieties for Resistance to Angoumois Grain Moth, Sitotroga cerealella (Olivier) (Lepidoptera: Gelechiidae) Nuclear Institute for Agriculture and Biology Pakistan J. Zool., vol. 38(1), pp. 7-10.

Teetes G, Reddy K, Leuschner K, House R. 1995. Manuel d'identification des insectes nuisibles au sorgho. Bulletin d'information $n^{\circ} 12$. Patancheru (Inde) : ICRISAT,

Teugwa, MC, Piam G, Tane P, Amvam Zollo PR. 2002. Activité insecticide des extraits d'Ageratum haustanianum, de Clausena anisata et de Cratan macrastachyus sur la bruche du niébé (Vigna unguiculata Walp). Faad-Africa, $4 \mathrm{p}$.

Togola A, Nwilene F, Chougourou CD, Agunbiade TA. 2010. Présence, population et dégâts de l'alucite des céréales Sitotroga cerealella sur les stocks de riz au Bénin 63p.

Tunç I, Berger BM, Erler F, Dagli F. 2000. Ovicidal activity of essential oils from five plants against two stored-products insects. J. Stored Prod. Res., 36, 161-168.

Vincent C, Hallman G, Panneton B, Fleurat-Lessard F. 2003. Management of Agricultural Insects with Physical Control Methods. Annu. Rev. Entomol., 48: 261-281.

Vowotor KA, Meikle WG, Ayertey JN, Markham RH. 2005. Distribution of and association between 
Adjalian et al. J. Appl. Biosci. 2014. État et perspectives de lute contre Sitotroga Cerealella (Olivier, 1789) de prédateur des céréales au Benin

the larger grain borer Prostephanus truncatus (Horn) (Coleoptera: Bostrichidae) and the maize weevil Sitophilus zeamais Motschulsky (Coleoptera: Curculionidae) in maize stores. J. Stored Prod. Res., 41, 498-512.
Zil'berg LP, Filipchuk IM. 1976. Applicator for the distribution of Trichogramma. Zashchita Rastenii, No. 9:20-21. 